\title{
Infiltración anestésica periprostática para biopsia de próstata por punción: estudio randomizado, doble ciego y controlado con placebo
}

\author{
Gonzalo Valero, EU Roxana González
}

Servicio de Urología. Hospital Regional de Rancagua. Chile.

Actas Urol Esp 2005; 29 (6): 550-552

\section{RESUMEN}

INFILTRACION ANESTESICA PERIPROSTATICA PARA BIOPSIA DE PROSTATA POR PUNCION: ESTUDIO RANDOMIZADO, DOBLE CIEGO Y CONTROLADO CON PLACEBO

Estudio prospectivo, randomizado, doble ciego y controlado con placebo evaluando la efectividad de la infiltración periprostática de lidocaína en disminuir el dolor de la biopsia por punción. En un período de trece meses, 115 pacientes fueron randomizados para recibir $10 \mathrm{ml}$ de lidocaína al $1 \%(\mathrm{n}=60)$ o suero fisiológico ( $\mathrm{n}=55$ ). Evaluando el dolor mediante escala visual análoga (0-10), el primer grupo refirió dolor promedio de 3,83 y el segundo de 6,87 , siendo esta diferencia significativa $(\mathrm{p}<0,005)$. No hubo complicaciones derivadas del anestésico. La infiltración periprostática es fácil de realizar, no presenta complicaciones y es efectiva en reducir el dolor del procedimiento. Debiera ser usada en forma rutinaria durante biopsia por punción.

Palabras claves: Lidocaína. Biopsia prostática. Dolor.

\section{ABSTRACT}

PERIPROSTATIC ANAESTHESIC INFILTRATION FOR PROSTATIC BIOPSY: A PROSPECTIVE, RANDOMIZED, DOUBLE BLIND AND PLACEBO-CONTROLLED STUDY

A prospective, randomized, double blind and placebo-controlled study to evaluate the effectiveness of periprostatic infiltration with lidocaine to reduce pain of prostatic biopsy. In a thirteen months period of time, 115 patients were randomized to receive $10 \mathrm{ml}$ of lidocaine $1 \%(n=60)$ or saline $(n=55)$. Evaluating the pain with visual analogue scale (0-10), the first group referred average pain of 3,83 and the second group of 6,87 , being this difference clearly significant $(\mathrm{p}<0,005)$. There were not complications from anesthesic puncture. The periprostatic infiltration is easy to perform without complications and it is effective in reducing the pain of this procedure. It should be used as a routine procedure in prostatic biopsy.

Keywords: Lidocaine. Prostatic biopsy. Pain.

$\mathrm{L}$ a biopsia prostática realizada mediante ultrasonido se ha convertido en el método de elección para el diagnóstico de cáncer prostático. Como todo procedimiento cruento produce angustia, dolor y molestias para el paciente. Varios estudios muestran que entre un 65 y $90 \%$ de los pacientes presentan dolor moderado y hasta un $20 \%$ de ellos no se repetirían el examen con la misma anestesia $^{1-3}$. El dolor, ansiedad y malestar es proporcional al número de punciones realizadas y en la actualidad existe un grado de consenso en que el número de biopsias debiera ser cercano a diez.

En un intento por disminuir estas molestias, varios autores han publicado trabajos utilizan- do sedación, anestésicos locales en forma de gel intrarectal o infiltración periprostática. Los resultados de esos reportes son contradictorios ya que algunos muestran poca o nula efectividad de los anestésicos locales. Existen estudios prospectivos y randomizados que han demostrado la efectividad de la lidocaína periprostática en disminuir el dolor del procedimiento ${ }^{4-8}$. De los anteriores, sólo uno es doble ciego y controlado con placebo ${ }^{7}$. Por lo anterior, el objetivo de este trabajo es confirmar los hallazgos de los mencionados estudios, aportando un estudio también prospectivo, randomizado y controlado con placebo. 


\section{MATERIAL Y METODO}

Previa aprobación por el Comité de Ética del hospital, 115 pacientes fueron enrolados para el estudio entre Junio de 2003 y Agosto de 2004. Todos los pacientes fueron informados en forma verbal y escrita acerca del procedimiento, firmando un consentimiento especial. La randomización fue realizada por la enfermera de nuestro Servicio, recibiendo el primer grupo de 60 pacientes 10 cc de lidocaína al $1 \%$ y el segundo grupo de 55 pacientes 10 cc de solución fisiológica.

La profilaxis antibiótica fue realizada con ciprofloxacino $500 \mathrm{mg}$ cada 12 horas un día antes del procedimiento y manteniéndolo por 48 horas después de la biopsia. En la noche previa al paciente también se le puso un enema evacuante. Ningún paciente estaba con anticoagulación o tomando antiagregantes plaquetarios.

La ecografia transrectal fue hecha con un transductor endocavitario de $7,5 \mathrm{MHz}$ y para la infiltración se ocupó una aguja $18 \mathrm{G}$, inyectando $3 \mathrm{ml}$ a cada lado de la base prostática en la unión con la vesícula seminal y el resto de la solución se colocó en el ápex entre la fascia de Dennonvillier y la cápsula prostática. La posición de la aguja se confirmó debido a la falta de resistencia para la infiltración y la visualización directa de la aparición de la pápula en las zonas mencionadas. Posterior a esto se procedió a efectuar las mediciones de la glándula ocupando unos 3 a 5 minutos, lo cual corresponde al tiempo de latencia del anestésico. Las biopsias fueron hechas en forma randomizada y por sextantes con un número promedio de muestras entre 8 y 10 . Para la punción prostática se utilizó una aguja 18G y la pistola ProMag 2.2 (Manatech Ltda., UK). Posterior al procedimiento se evaluó el dolor mediante una escala visual análoga, que se gradúa de 0 (sin dolor) hasta 10 (el dolor más intenso nunca antes experimentado).

Para el análisis estadístico se ocupó la prueba $\mathrm{t}$ de Student no pareado, con un $\mathrm{p}<0,05$ como valor significativo.

\section{RESULTADOS}

Durante un período de 13 meses, se reclutaron 115 pacientes, recibiendo 60 anestésico local y 55 suero fisiológico. Dos pacientes fueron excluidos del estudio por analfabetismo (imposibilidad de leer la escala visual análoga). Ambos grupos fueron semejantes en cuanto a edad, volumen prostático, valor de PSA y número de muestras. (Tabla 1).

\section{Tabla 1}

Características Generales de la Serie

\begin{tabular}{lcc}
\hline Variables & Anestésico local & Placebo \\
\hline Número de pacientes & 60 & 55 \\
Edad (promedio) & $68,9 \mathrm{años}$ & $67,7 \mathrm{años}$ \\
PSA (mediana) & $8,87 \mathrm{ng} / \mathrm{cc}$ & $9,2 \mathrm{ng} / \mathrm{cc}$ \\
$\begin{array}{l}\text { Volumen prostático } \\
\text { (promedio) }\end{array}$ & $64,1 \mathrm{cc}$ & $61,6 \mathrm{cc}$ \\
$\begin{array}{l}\text { Número de biopsias } \\
\text { (promedio) }\end{array}$ & 9,9 & 9,8 \\
\hline
\end{tabular}

El número de biopsias tomadas varió entre 8 y 10 con un promedio de 9,3. En el 88\% (101/110) de los pacientes se tomaron 10 muestras.

El primer grupo presentó un promedio de dolor significativamente menor que el grupo tratado con suero fisiológico, con un promedio de 3,83 y 6,87 respectivamente ( $\mathrm{p}<0,001$ ) (Fig. 1). No hubo complicaciones derivadas de la infiltración de anestesia local. Un paciente del segundo grupo debió ser hospitalizado por una infección urinaria complicada.

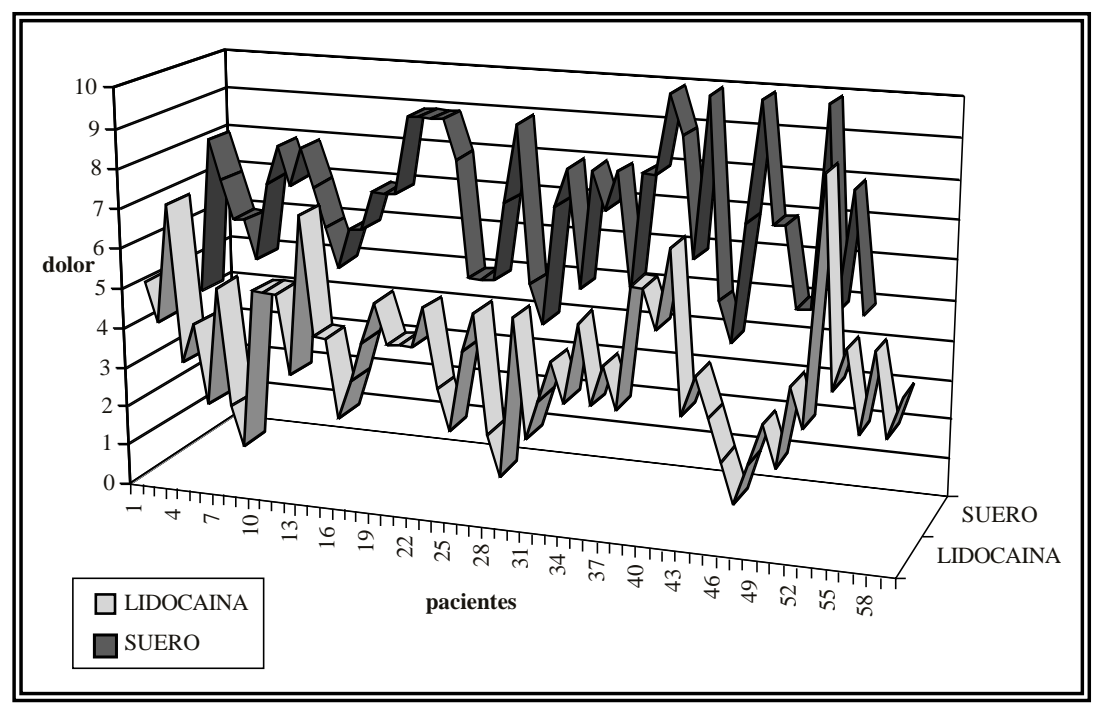

FIGURA 1. Evaluación del dolor para ambos grupos de pacientes. Valor $p<0,001$. 


\section{DISCUSION}

Para lograr una buena anestesia, se deben bloquear todas las vías aferentes que llevan el estímulo doloroso desde la glándula prostática hasta la médula. Estas vías nerviosas se ubican junto a los paquetes vasculares que corren en forma lateral a la próstata. En este sentido, la infiltración de los pedículos a nivel lateral y basal debiera ser el método más efectivo en disminuir o bloquear el dolor del procedimiento como lo han demostrado algunos autores $^{4,5} \mathrm{y}$ ha sido el objetivo del presente estudio. Se han usado otras formas de administración del anestésico como gel intrarectal con resultados no favorables y cuando se ha comparado el gel con la infiltración periprostática, esta última ha demostrado ser superior ${ }^{9-11}$. Al igual que otros reportes, coincidimos en que las punciones de la zona apical son más dolorosas que la media o basal, por este motivo también en esta experiencia usamos la inyección de $3 \mathrm{ml}$ de lidocaína en el ápex.

Con respecto a las eventuales complicaciones del anestésico local, como la inyección intravascular, ésta no ocurrió y creemos que se debe al pequeño grosor de los vasos de los haces vasculares. Donde sí puede existir riesgo es en la zona apical donde hay un riesgo potencial de inyectar el anestésico en el complejo dorsal, sin embargo éste es bastante fácil de identificar por sobre la uretra y más aun cuando se cuenta con el Doppler color. Los pacientes que fueron posteriormente intervenidos para una cirugia radical no presentaron dificultades técnicas para la disección de la glándula como fibrosis o tejido inflamatorio.

Hay reportes que han mostrado que la biopsia prostática produce más dolor e incomodidad en pacientes más jóvenes ${ }^{12}$. En este estudio no hubo relación entre la evaluación del dolor ni con la edad de los pacientes ni tampoco con el volumen de la glándula.

Para poder mejorar el rendimiento de los resultados, dispusimos en el protocolo que el número de muestras fuera lo más regular posible para ambos grupos y es así como variaron entre 8 y 10 , realizando en la gran mayoría de los pacientes (88\%) 10 muestras. Lo anterior fue para hacer aun más a ambos grupos comparables, ya que si la cantidad total de muestras era diferente, se podía inferir que la diferencia entre ambos grupos era consecuencia de esto y no por el efecto del anestésico local.
En trabajos posteriores, será de interés investigar la forma de administración (técnica de la punción) y las dosis del anestésico local a utilizar para lograr un buen efecto analgésico.

Como conclusión podemos establecer que la infiltración periprostática con anestésico local reduce en forma significativa el dolor y que debido a su fácil administración y bajos riesgos debiera ser de uso rutinario en la biopsia prostática ecoguiada.

\section{REFERENCIAS}

1. Clements R, Aideyan OU, Griffiths GJ, Peeling WB. Side effects and patient acceptability of transrectal biopsy of the prostate. Clin Radiol 1993;47(3):174-176.

2. Collins GN, Lloyd SN, Hehir M, McKelvie GB. Multiple transrectal ultrasound-guided prostatic biopsies-true morbidity and patient acceptance. Br J Urol 1993;71(4):460-463.

3. Irani J, Fournier F, Bon D, Gremmo E, Dore B, Aubert J. Patient tolerance of transrectal ultrasound-guided biopsy of the prostate. Br J Urol 1997;79(4):608-610.

4. Nash PA, Bruce JE, Indudhara R, Shinohara K. Transrectal ultrasound guided prostatic nerve blockade eases systematic needle biopsy of the prostate. J Urol 1996;155:607-609.

5. Alavi AS, Soloway MS, Vaidya A, Lynne CM, Gheiler EL. Loca anaesthesia for ultrasound guided prostate biopsy: a prospective randomised trial comparing 2 methods. J Urol 2001;166:1343-1345.

6. Seymour H, Perry MJA, Lee-Elliot C, Dundas D, Patel U. Pain after transrectal ultrasonography-guided prostate biopsy: the advantages of periprostatic local anaesthesia. BJU Int 2001;88:540-544.

7. Addla S, Adeyoju A, Wemyss-Holden G, Neilson D. Local Anaesthetic for Transrectal Ultrasound-Guided Prostate Biopsy: A Prospective, Randomized, Double Blind, PlaceboControlled Study. Eur Urol 2003; 43: 441-443.

8. Kaver I, Mabjeesh N, Matzkin H. Randomized prospective study of periprostatic local anesthesia during transrectal ultrasound-guided prostate biopsy. Urology 2002;59:405408.

9. Issa MM, Bux S, Chun T, Petros JA, Labadia AJ, Anastasia K, et al. A randomized prospective trial of intrarectal lidocaine for pain control during transrectal prostate biopsy: the Emory University experience. J Urol 2000;164:397.

10. Lynn NNK, Collins GN, Brown SCW, O'Reilly PH. Periprostatic nerve block gives better analgesia for prostatic biopsy. BJU Int 2002;90:424-426

11. Rodríguez A, Kyriakou G, Leray E, Lobel B, Guillé F. Prospective Study Comparing Two Methods of Anaesthesia for Prostate Biopsies: Apex Periprostatic Nerve Block versus Intrarectal Lidocaine Gel: Review of the Literature. Eur Urol 2003; 44:195-200

12. Rodriguez LV, Terris MK: Risks and complications of transrectal ultrasound guided prostate needle biopsy: a prospective study and review of the literature. J Urol 1998;160:2115-2120.

Dr. G. Valero

Servicio de Urología

Hospital Regional de Rancagua

Chile

e-mail: gonzalovalero@terra.cl

(Trabajo recibido el 15 febrero de 2005) 